\title{
Downregulation of discoidin domain receptor 2 in A375 human melanoma cells reduces its experimental liver metastasis ability
}

\author{
IKER BADIOLA $^{1,2}$, PATRICIA VILLACÉ ${ }^{1}$, IRATXE BASALDUA ${ }^{2}$ and ELVIRA OLASO ${ }^{2}$ \\ ${ }^{1}$ Innoprot S.L., Parque Tecnológico de Zamudio, Ed. 502, Derio 48160; ${ }^{2}$ Department of Cell Biology, \\ School of Medicine, University of the Basque Country, B ${ }^{\circ}$ Sarriena s/n, Leioa 48940, Spain
}

Received January 19, 2011; Accepted February 28, 2011

DOI: $10.3892 /$ or.2011.1356

\begin{abstract}
Discoidin domain receptors (DDR1 and DDR2) are tyrosine kinase receptors for fibrillar collagen implicated in postnatal development, tissue repair, and primary and metastatic cancer progression. While DDR 1 has been described in tumor cells, DDR2 has been localized in the tumor stroma, but its presence in the tumor cells remains unknown. The aim of this study was to elucidate the role of DDR2 signaling in tumor cells during hepatic metastasis progression. DDR2 expression and phosphorylation in cultured human A375 melanoma cells was documented by Western blot analysis. A375 cells were stably transfected with a small interfering RNA (siRNA) against DDR2 and two clones were selected: A375R2-70 and A375R2-40, with 70 and $40 \%$ of the DDR2 protein expression respectively, compared to mock-transfected cells (A375R2-100). Development of experimental liver metastasis by intrasplenic inoculation of A375R2-70 and A37R2-40 clones was reduced by 60 and $75 \%$, respectively, measured as tumor volume, compared to livers injected with A375R2-100 cells. Accordingly, A375R2-70 and A37R2-40 clones showed reduced in vitro gelatinase activity and JNK phosphorylation, compared to mock transfected cells, with maximal inhibition in A375R2-40. Additionally, A375 melanoma, SK-HEP hepatoma and HT-29 colon carcinoma human cell lines transiently transfected with siRNA against DDR2 also showed reduced proliferation and migration rates compared to mock-transfected ones. In conclusion, DDR2 promotes A375 melanoma metastasis to the liver and the underlying mechanism implicates regulation of metalloproteinase release, cell growth and chemotactic invasion of the host tissue.
\end{abstract}

Correspondence to: Dr Elvira Olaso, Department of Cell Biology, School of Medicine, University of the Basque Country, $\mathrm{B}^{\circ}$ Sarriena s/n, Leioa 48940, Spain

E-mail: elvira.olaso@ehu.es

Key words: melanoma, discoidin domain receptor 2, matrix metalloproteinases, collagen receptors, liver metastasis

\section{Introduction}

The lack of knowledge on the molecular pathways regulating liver metastasis by primary melanoma tumors is a major hurdle for more efficient, targeted oriented treatment (1). Tyrosine kinase receptors (TKRs) have been related to cancer progression and malignancy (2). TKR union to a specific ligand provokes receptor oligomerization and conformational changes that activate the catalytic domain tyrosine kinase (3), induces autophosphorylation at tyrosine residues and initiates a signalling cascade (4) that elicits key cell responses such as proliferation, differentiation, apoptosis or transformation (3-5). Discoidin domain receptors (DDRs) are a family of TKRs that signal in response to collagen type I instead of soluble growth factors (6). DDR1 is expressed in tumor cells from lung cancer, leukemia and hepatocelluar carcinoma (7-9) while DDR2 has been described in the stromal cells surrounding epithelial tumors (10). DDR receptors present a lower phosphorylation pattern than the other TKRs, which may relate this receptor with chronic diseases (11). Also, DDR receptors mediate matrix metalloproteinase (MMP) production, and cell migration and proliferation in several onsets of experimental and human tissue remodelling $(12,13)$.

In this study, we describe DDR2 expression and signalling in several human cell lines. We also describe DDR2 implication in liver metastasis progression by A375 human melanoma mediated by metalloproteinase production and cell proliferation and migration.

\section{Materials and methods}

Cell culture. Human melanoma A375 cell line was cultured at $37^{\circ} \mathrm{C}$, in $\mathrm{CO}_{2} 5 \%$ with RPMI medium supplemented with penicillin, amphotericin A and streptomycin $(100 \mu \mathrm{g} / \mathrm{ml})$ and $10 \%$ of FBS. The human colon carcinoma HT29 cell line was cultured at $37^{\circ} \mathrm{C}$, in $\mathrm{CO}_{2} 5 \%$ in McCoy medium supplemented with penicillin, amphotericin A and streptomycin $(100 \mu \mathrm{g} /$ $\mathrm{ml}$ ) and $10 \%$ of FBS. The human liver carcinoma SK-HEP cell line was cultured at $37^{\circ} \mathrm{C}$, in $\mathrm{CO}_{2} 5 \%$ with DMEM medium supplemented with penicillin, amphotericin $\mathrm{A}$ and streptomycin $(100 \mu \mathrm{g} / \mathrm{ml})$ and $10 \%$ of FBS. Before the experiments, cells were detached by exposure to $4 \mathrm{mM}$ PBS-EDTA. The obtained cell suspension was centrifuged to remove cell 
debris and suspended in fresh medium to a final concentration of $10^{6}$ cells $/ \mathrm{ml}$.

Immortalized human hepatic stellate cell line LX2 was cultured at $37^{\circ} \mathrm{C}$, in $\mathrm{CO}_{2} 5 \%$ with DMEM medium supplemented with penicillin, amphotericin A and streptomycin $(100 \mu \mathrm{g} / \mathrm{ml})$ and $1 \%$ of FBS. Before the experiments the cells were detached by exposure to $0.05 \%$ Trypsin-EDTA. The obtained cell suspension was centrifuged to remove cell debris and suspended in fresh medium to a final concentration of $10^{6}$ cells $/ \mathrm{ml}$.

Cell transfection. The specific small interfering RNA (siRNA) hairpins against DDR2 (AAC-CUG-AUG-ACCUGA-AGG-AGU) and the non-silencing mock control (AAU-UCU-CCG-AAC-GUG-UCA-CGU) fragments were ligated with a 5'MseI site introduced by polymerase chain reaction (PCR) into pSuper plasmid. All the DNA sequences derived from PCR were subcloned into the EcoRI site to pLEN for stable transfection into cell lines using lipofectamine (Invitrogen). Cells were allowed to grow under G418 selection, and individual colonies were selected and screened for silencing efficiency by Western blot detection of DDR2. Two clones were selected: one with $70 \%$ of DDR2 protein expression respect to the mock (A375R2-70) and other one with $40 \%$ of expression (A375R2-40). Also, a mock-transfected clone (A375R2-100) with the same DDR2 expression levels as untransfected cells was created.

For the transient transfection the A375, HT29 and SK-HEP cell lines were incubated for $4 \mathrm{~h}$ with the mixture of lipofectamine and siRNA against DDR2. After $72 \mathrm{~h}$ of transfection, the silencing efficiency was measured by RT-PCR.

$R T-P C R$. Total RNA from cells was isolated using TRIzol Reagent (Invitrogen) according to the manufacturer's protocol. Total RNA $(1 \mu \mathrm{g})$ was reverse-transcribed to cDNA. Briefly, $1 \mu \mathrm{g}$ of total RNA, $1 \mu \mathrm{l}$ of $10 \mathrm{mM}$ dNTPs, $1 \mu \mathrm{l}$ of retrotranscription enzyme (Takara) and DEPC water were incubated for $25 \mathrm{~min}$ at $70^{\circ} \mathrm{C}$. Next, the polymerase chain reaction for DDR2 and GAPDH like loading control were performed. cDNA $(1 \mu \mathrm{g})$ was added to $2 \mu \mathrm{l} 10 \mathrm{X}$ buffer mixture (BioRad, CA, USA), $4 \mu \mathrm{l}$ of $25 \mathrm{mM} \mathrm{Mg} \mathrm{Cl} 2,2 \mu \mathrm{l}$ of $100 \mathrm{mM}$ dNTPs, $1 \mu \mathrm{l}$ of $500 \mathrm{mM}$ primer mix (DDR2: Forward 5'-TGG-CAC-CCA-CAA-CCT-ATG-ATC-3'; Reverse: 5'-GGT-GAT-GAG-GAG-CGG-TTA-TTG-3'. GAPDH: Forward: 5'-GCG-CCT-CAA-GGT-GTT-GGA-T-3'; Reverse: 5'-GCC-CCA-GGT-AAG-CAA-ACT-TTC-3') and $1 \mu \mathrm{l}$ of polymerase enzyme (Bio-Rad) completed with DEPC water for total of $20 \mu \mathrm{l}$. The mixture was incubated for $5 \mathrm{~min} 95^{\circ} \mathrm{C}$ for denaturing procedure, followed by 35 cycles of $95^{\circ} \mathrm{C}$ for $30 \mathrm{sec}, 51^{\circ} \mathrm{C}$ for $30 \mathrm{sec}$ and $10 \mathrm{~min}$ for a final extension at $72^{\circ} \mathrm{C}$. The product was analyzed in an agarose gel and the resultant bands were quantified with the Quantity One software (Bio-Rad).

Western blotting. Cell lysates were obtained by adding $100 \mu 1 / 10^{6}$ cells of lysis buffer to cell cultures. Cell lysates were immunoprecipitated with anti-DDR2 antibody (sc-7555 Santa Cruz) at $4^{\circ} \mathrm{C}$ overnight and incubated with Protein A Sepharose for $2 \mathrm{~h}$ at $4^{\circ} \mathrm{C}$. Then, immunoprecipitates were centrifuged 3 times at $50 \mathrm{x}$ g for $10 \mathrm{~min}$ each in lysis buffer. Electrophoresis in 4-6\% polyacrylamide gels was performed under denaturing conditions. Prior to use, anti-DDR2 antibody was diluted in blotting blocking buffer 1:1000, and anti-phosphotyrosine antibody (Cell Signaling) was diluted 1:2000. Membranes were exposed to antibody solution for $1 \mathrm{~h}$ followed by appropriate secondary antibodies conjugated with HRP (1:10000 dilution in blotting blocking buffer). Hyperfilm (Amershan Bioscience) was used for the membrane and specific band analyses with the Quantity One software (Bio-Rad).

Liver metastasis model. Hepatic metastases were produced by intrasplenic injection of $1 \times 10^{6}$ A 375 cells into anesthetized C57BL/6J-Hfn11 nude mice as previously reported (14). Mice were sacrificed 30 days later. All animals received humane care according to the criteria outlined in the 'Guide for the Care and Use of Laboratory Animals' prepared by the National Academy of Sciences and published by the National Institutes of Health.

Liver sections $(10 \mu \mathrm{m})$ fixed in cold acetone were processed for hematoxylin/eosin (H\&E) staining $(n=12)$ and the relative volume occupied by the tumor was analyzed under the microscope.

Migration assay. The migration assay was carried out in modified Boyden chambers. The human cancer cell lines were cultured onto $8-\mu \mathrm{m}$ pore membranes in RPMI medium. Collagen (10 nM) (Becton-Dickinson) diluted in RPMI medium was added in the lower compartment. After $4 \mathrm{~h}$ of incubation, chambers were stained with H\&E and cells that were in the bottom of the membrane were counted under the microscope at $\mathrm{x} 20$ (12).

Proliferation assay. Subconfluent cells were cultured for $24 \mathrm{~h}$ in the presence or absence of collagen type I (10 nM). After $24 \mathrm{~h}$ incubation $100 \mu \mathrm{l}$ of MTT solution $(0.5 \mathrm{mg} / \mathrm{ml})$ (15) was added and allowed to react for $1 \mathrm{~h}$ at $37^{\circ} \mathrm{C}$ in a $5 \%$ $\mathrm{CO}_{2}$ incubator. Reaction was stopped by addition of DMSO. Absorbance of the converted dye in living cells was measured at a wavelength of $650 \mathrm{~nm}$. Proliferation index was calculated as a percentage of initial control cell concentration.

Zymography. The cells were cultured to subconfluence in serum-free media and incubated overnight with or without soluble collagen type I (10 nM). Then media were collected, centrifuged at maximum speed to remove cell debris and stored at $-80^{\circ} \mathrm{C}$ for further use. Zymography was performed as previously reported (11). In brief, gelatin-containing gels were loaded with samples diluted in loading buffer without $\beta$-mercaptoethanol and the electrophoresis was run $10 \mathrm{~min}$ at $120 \mathrm{mV}$ followed by $1 \mathrm{~h}$ at $200 \mathrm{mV}$. Then, the gels were incubated for $12 \mathrm{~h}$ in the incubation buffer $(1 \mathrm{M} \mathrm{CaCl}, 1 \mathrm{M}$ Tris- $\mathrm{HCl}$, $0.25 \mathrm{M} \mathrm{NaN}_{3}$ and $5 \mathrm{mM} \mathrm{ZnCl}_{2}$ ) at $37^{\circ} \mathrm{C}$. Finally, the gels were stained with a Coomassie Blue solution. Quantity One software (Bio-Rad) was used to measure the gelatinase activity.

ELISA assay. 20,000 human melanoma A375 cells were cultured in RPMI medium in two 96-wells plates. One plate was used to measure the total protein and the other one the phosphorylated protein. In each, half the plate was incubated with collagen $(10 \mathrm{nM})$ and the other half in basal conditions. 
A

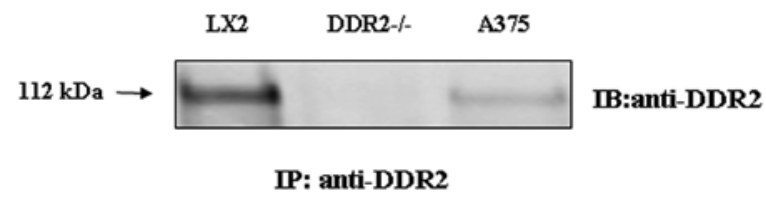

B

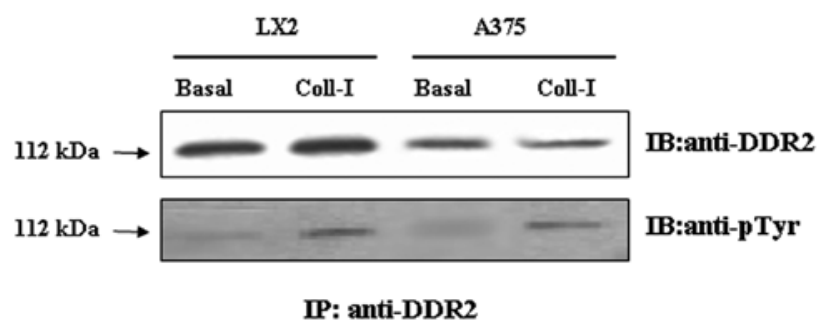

Figure 1. DDR2 expression and phosphorylation in the human A375 melanoma cell line. (A) Immunoprecipitation for DDR2 and further Western blotting against DDR2 in human A375 melanoma cell lysates were performed. An expression band of $112 \mathrm{kDa}$ was detected. (B) Immunoprecipitation for DDR2 and further Western blotting against phosphorylated DDR2 of A375 human melanoma cell line were performed. After stimulation with collagen for $120 \mathrm{~min}$, the expression band of phosphorylation was observed. LX2 hepatic stellate cells were used as positive and DDR2 knock-out skin fibroblasts were used as negative control.

The cells were fixed and incubated with the anti-protein (JNK) antibody and with the anti-phosphoprotein. Then the wells were washed with PBS and incubated for $1 \mathrm{~h}$ at room temperature with the secondary antibody HRP conjugated. The result was read at $650 \mathrm{~nm}$.

Statistical analysis. Statistical results refer to mean \pm SD. Statistical analysis was performed by SPPS statistical software for Microsoft Windows, release 6.0 (Professional Statistics, Chicago, IL). Individual comparisons were made with Student's two-tailed, unpaired t-test using a P-value of 0.05 as criterion of statistical significance. We use the non-parametric Spearman correlation coefficient to compare the results and calculate if the differences were statistically significant. Each experiment was repeated three times.

\section{Results}

A375 human melanoma cells express functional DDR2 that becomes phosphorylated by soluble Collagen type I. Expression levels of DDR2 protein in the human A375 melanoma cell line were determined by Western blotting using anti-DDR2 antibody (sc-7555). Immortalized human LX2 hepatic stellate cells (16) were used as positive control and DDR2 knock-out skin fibroblasts (12) were used as negative controls. As shown in Fig. 1A, DDR2 was observed in the A375 lysates as a $112 \mathrm{kDa}$ band.

DDR2 signaling initiates as receptor phosphorylation (14). Addition of soluble collagen type I to subconfluent cultures of A375 cells resulted in DDR2 phosphorylation, as observed by receptor immunoprecipitation with sc- 7555 followed by immunoblot analysis for phosphotyrosine (Fig. 1B).
$\mathbf{A}$

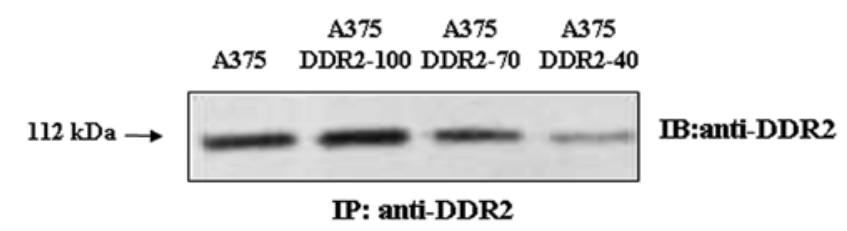

B

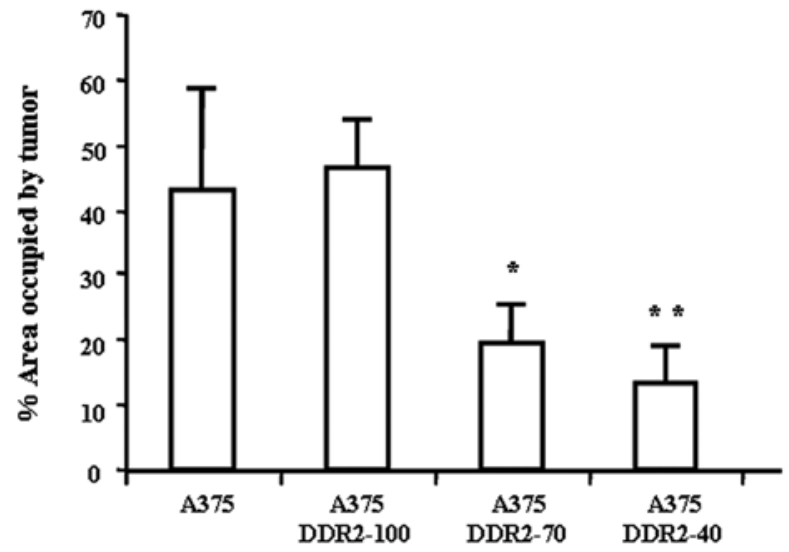

Figure 2. (A) Western blot analysis of DDR2 siRNA transfection efficiency. Cells were submitted to DDR2 immunoprecipitation followed by Western blot analysis of DDR2 expression to estimate the silencing level. The mock transfected cell line A375R2-100 was considered as 100\% DDR2 protein expressing. A375R2-70 and A375R2-40 cell clones expressed 70 and 40\% of DDR2 with respect to the control A375R2-100. (B) Experimental liver metastasis of A375, A375R2-100, A375R2-70 and A375R2-40 cell clones. Nude mice were injected intrasplenically with the A375 cell clones. After 30 days the mice were sacrificed and the liver processed and stained with hematoxylin/eosin. Percentage of liver volume occupied by metastases was analyzed as previously described (19). ${ }^{*} \mathrm{p}<0.05$ and ${ }^{* *} \mathrm{p}<0.001$ compared to mock transfected.

Downregulation of DDR2 expression in A375 cells reduces their experimental liver metastatic capacity in nude mice. Two A375 cell lines with stable silencing of DDR2 were created by transfection of a specific siRNA against the DDR2 mRNA. One selected clone expressed 70\% of DDR2 protein (A375R2-70) compared with the mock transfected cell line (A375R2-100), which expressed same levels as control. The other clone expressed $40 \%$ of DDR 2 protein (A375R240) with respect to control. Protein expression levels were measured by Western blotting. Silencing efficiency was tested after $72 \mathrm{~h}$ in culture (Fig. 2A).

To study the implication of DDR2 in the metastatic capabilities of human A375 melanoma cells, experimental liver metastasis was studied by intrasplenic inoculation of A375R2-100, A375R2-70 and A375R2-40 and control A375 cells into C57BL/6J-Hfn11nu nude mice. After 30 days, liver tissues were processed for immunohistochemistry and analyzed as described in Materials and methods. Liver metastatic foci developed in all treated nude mice. As shown in the histogram of Fig. 2B, percentage of liver tissue occupied by the tumor was reduced from $48 \%$ in mice injected with mock transfected A375R2-100 to $20 \%$ in mice inoculated with A375R2-70 and to $12 \%$ in mice inoculated with A375R2-40. The liver tissue area occupied by the A375 cell microtumors and by the mock transfected A375R2-100 cell injected mice 
$\mathbf{A}$

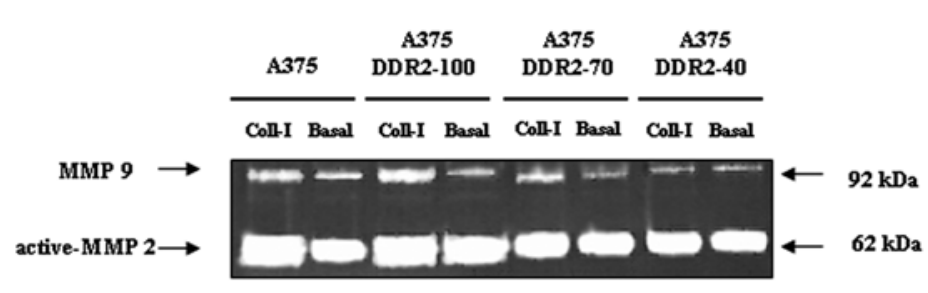

B
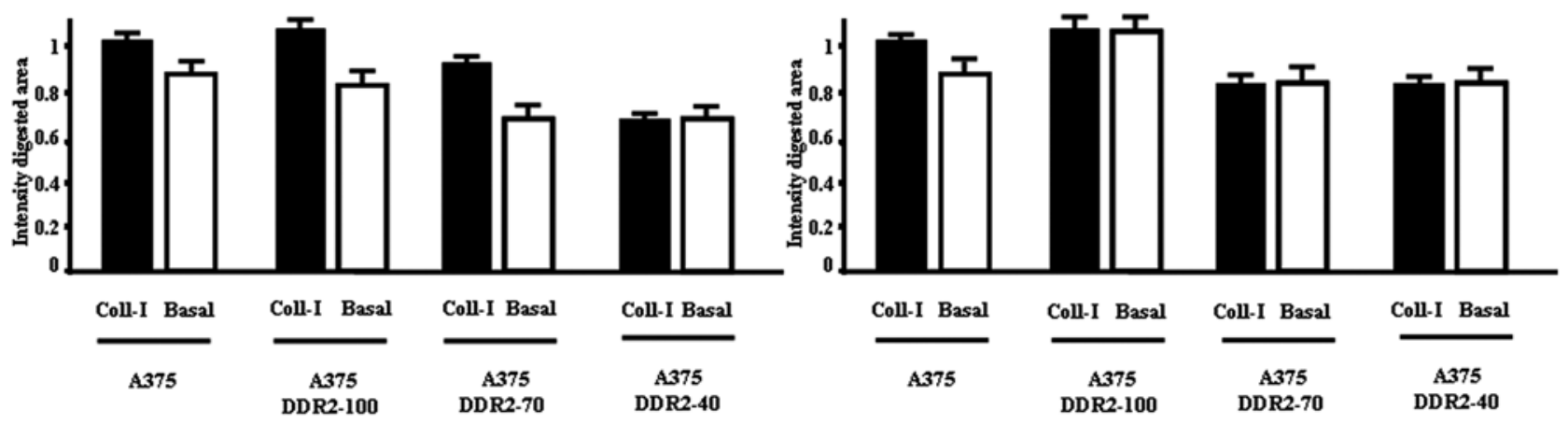

Figure 3. DDR silencing in A375 human melanoma cells reduces MMP activity. (A) A375, A375R2-100, A375R2-70 and A375R2-40 cell clones were cultured under basal conditions or in the presence of $10 \mathrm{nM}$ soluble collagen type I. Culture supernatant were collected and submitted to gelatine zymography as previously described (18). Two bands at $92 \mathrm{kDa}$ (MMP9) and $62 \mathrm{kDa}$ (MMP2) were observed for all samples. (B and C) Intensity of the area digested by MMP9 (B) and MMP2 (C) was measured using Quantity One software (Bio-Rad).

$\mathbf{A}$

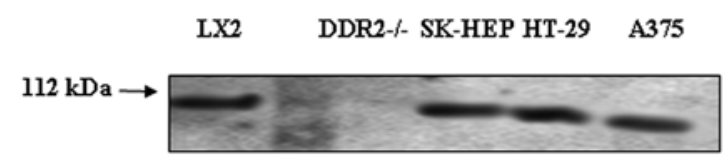

IB:anti-DDR2

IP: anti-DDR2

B

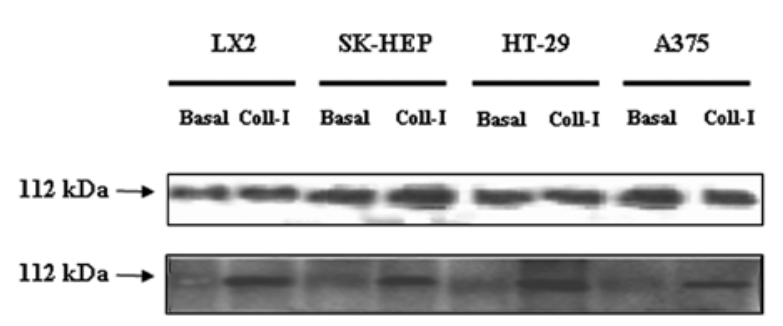

IB: anti-DDR2

IP: anti-DDR2

Figure 4. DDR2 expression and phosphorylation in human SK-HEP, HT-29 and A375 cancer cell lines. (A) Immunoprecipitation/Western blotting against DDR2 in human cancer cell line SK-HEP, HT-29 and A375 cell lysates were performed. An expression band (112 kDa) was detected in all the samples. DDR2 knock-out skin fibroblasts were used as negative control. (B) Immunoprecipitation/Western blotting for DDR2 and tyrosine phosphorylation were performed in SK-HEP, HT-29 and A375 cell lines. After stimulation with collagen for 120 min, an expression band corresponding to phosphorylated DDR2 was observed. LX2 hepatic stellate cells were used as a positive control.

were identical, indicating that the transfection process did not affect the metastatic capabilities of the A375 cells.

Downregulation of DDR2 expression correlates with reduced MMP9 and MMP2 activity in A375 melanoma cells. A375 cells were incubated for $120 \mathrm{~min}$ in serum-free media in the presence or absence of $10 \mathrm{nM}$ soluble collagen type I. Then, culture supernatants were analyzed by gelatin zymography. Two bands of $92 \mathrm{kDa}$ (corresponding to MMP9) and $62 \mathrm{kDa}$ (corresponding to active MMP2) were observed in all samples 

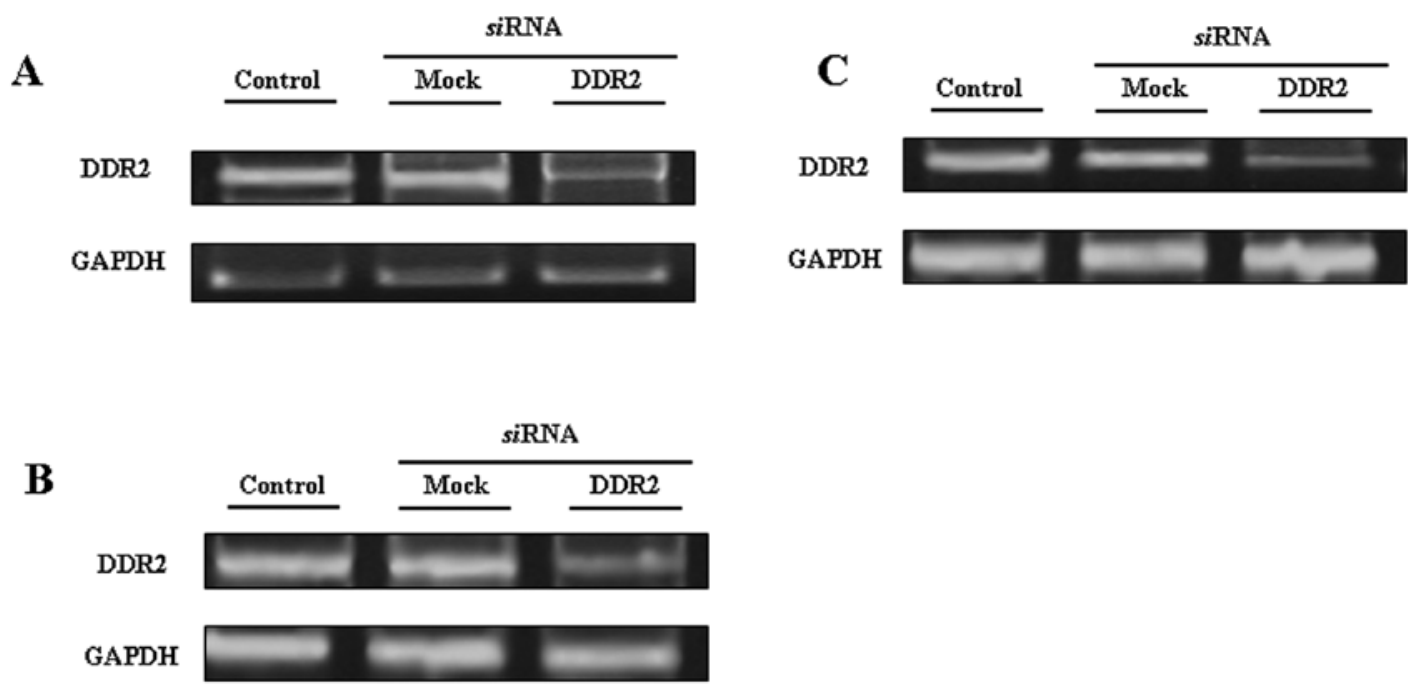

Figure 5. RT-PCR analysis of transient transfection of DDR2 siRNA on DDR2 mRNA expression. The human cancer cell lines SK-HEP (A), HT-29 (B) and A375 (C) were transiently transfected with siRNA against DDR2. The silencing efficiency was measured by RT-PCR and expressed relative to GAPDH mRNA expression levels. Silencing efficiency was measured comparing with DDR2 mRNA expression in mock transfected cells.

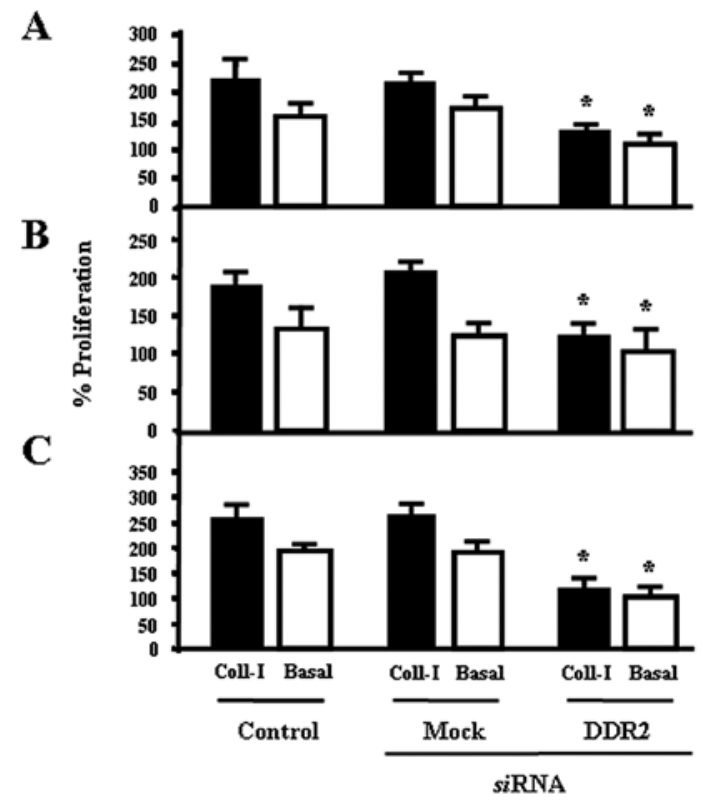

Figure 6. Downregulation of DDR2 protein expression in A375, SK-HEP and HT-29 human cancer cell lines reduces their in vitro proliferation capacity. Subconfluent cells were cultured in presence of collagen $(10 \mathrm{nM}$, black bars) or in the absence (white bars) for $24 \mathrm{~h}$. MTT assay was performed to measure the proliferative capacity of SK-HEP (A), HT-29 (B) and A375 (C) human cell lines with partially silenced DDR2 expression, compared to that of non-silenced ones. Statistically significant value * $p<0.05$.

(Fig. 3A). MMP activity was analyzed measuring the digested area. Addition of collagen type I in all A375 cell line cultures resulted in an average 20-25\% increase in their secreted MMP9 activity, compared to basal conditions, irrespective of their levels of DDR2 expression. MMP9 activity in the supernatants of collagen incubated A375R2-70 and A375R2-40 decreased by 15 and $25 \%$, respectively, compared to MMP activity in the mock-transfected A375R2-100 cells and the decrease was 22\% in both cell lines in basal conditions (Fig. 3B).
The clones showed no difference in MMP2 activity between incubation with or without collagen type I. Both A375R2-70 and A375R2-40 downregulated MMP2 activity compared to mock control at the same level, 20\% (Fig. 3C).

SK-HEP human hepatocarcinoma and HT29 human colon carcinoma express functional DDR2. DDR2 expression was also described in human SK-HEP hepatocarcinoma and HT-29 colon carcinoma cancer cell lines (Fig. 4A). Human LX2 cells (16) were used as positive control and DDR2 knock-out skin fibroblasts (12) were used as negative control. DDR2 protein expression band was detected at $112 \mathrm{kDa}$. The functionality of the receptor was assessed as tyrosine phosphorylation in response to soluble collagen type I (Fig. 4B), as previously described for A375 (Fig. 1B).

Downregulation of DDR2 expression in human cancer cell lines reduces their in vitro proliferation and migration capacity. MMP activity has been correlated with tumor cell proliferation and migration capacities (17). SK-HEP, HT29 and A375 human cancer cell lines were transiently transfected with siRNA against DDR2 to partially silence its protein expression (Fig. 5A-C). After $72 \mathrm{~h}$ of culture, silencing efficiencies $>50 \%$ were observed by RT-PCR with an average $65 \%$ for SK-HEP, 55\% for HT-29 and 52\% for A375, compared to their respective mock transfected and untransfected cells. Each cell lines and their corresponding silenced pools were cultured in the presence or absence of soluble type I and cell proliferation was assayed by the MTT method $48 \mathrm{~h}$ after silencing (Fig. 6A-C). Under basal culture conditions (no collagen added), the reduction of the proliferation capacity was $44 \%$ for SK-HEP, $15 \%$ for HT-29 and 35\% for A375, compared to untransfected cells. Interestingly, incubation with DDR2 ligand soluble collagen type I induced cell proliferation in mock/untransfected cells while inhibited proliferation in DDR2 silenced ones down to 55\% for SK-HEP, 45\% for HT-29 and 55\% A375 compared to untransfected cells. 


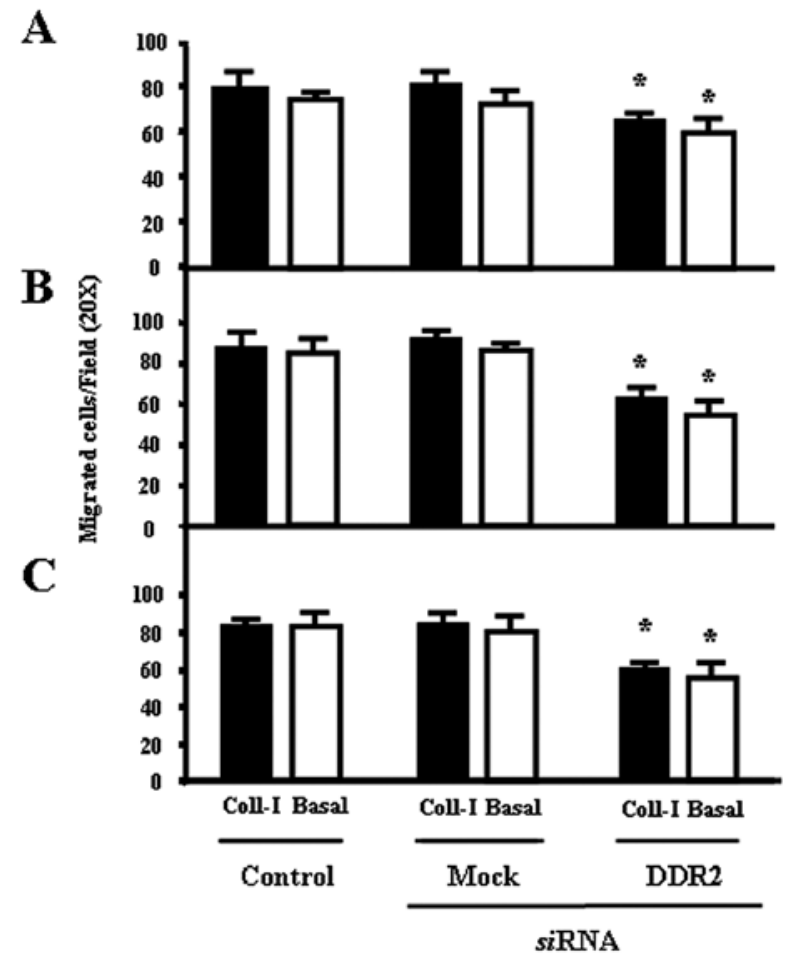

Figure 7. Downregulation of DDR2 protein expression in A375, SK-HEP and HT-29 human cancer cell lines reduces their in vitro migration capacity. A migration assay for DDR2 partially silenced and non-silenced SK-HEP (A), HT-29 (B) and A375 (C) was performed by culturing the cells in modified Boyden chamber and allowing them to migrate across its porous membrane in response to collagen type I gradient. The cells were cultured in the presence (black bars) or the absence (white bars) of fibrillar collagen on the membrane. After $24 \mathrm{~h}$, the cells were fixed and dyed with hematoxylin/ eosin to count the number of migrated cells. Statistically significant value ${ }^{*} \mathrm{p}<0.05$.

Migration rates did not significantly change in response to collagen type I compared to basal media (Fig. 7A-C). In basal conditions the reduction was $15 \%$ for SK-HEP, $30 \%$ for HT-29 and $22 \%$ for A375 compared to the controls and when the DDR2 ligand collagen was added, the reduction was $22 \%$ for SK-HEP, $35 \%$ for HT-29 and $27 \%$ for A375.
DDR2 signaling in A375 cells in response to soluble collagen type I involves JNK phosphorylation. An ELISA assay was performed to analyze JNK pathway activation following collagen phosphorylation of control A375 and silenced A375R2-70 and A375R2-40 clones (Fig. 8). As in the previous proliferation and migration experiments, cells were cultured under either basal conditions or in the presence of soluble collagen type I.

JNK protein (75\%) was already phosphorylated under basal conditions in control A375R2-100 cells. Addition of collagen type I rendered them with a $100 \%$ of JNK protein phosphorylated. JNK phosphorylation in DDR2 downregulated cell lines was reduced by $>50 \%$ under both basal conditions and in the presence of collagen for A375R2-70 and 70\% for A375R2-40, suggesting that JNK phosphorylation is an event downstream of DDR2 signaling.

\section{Discussion}

Our study demonstrates for the first time the presence of functional DDR2 in human A375 melanoma, SK-HEP hepatocarcinoma and HT-29 colon carcinoma human cell lines. We further show that DDR2 signaling in these tumor cell lines mediates key aspects of tumor progression such as secretion of metalloproteinases, migration and proliferation. We also characterize JNK phosphorylation as a downstream event in the DDR2 signaling pathway in these cell lines.

We previously demonstrated DDR2 implication in liver fibrosis and skin wound-healing $(11,12,17)$. In this study, we utilized the siRNA technique to correlate DDR2 expression in A375 melanoma cell clones with their hepatic metastatic potential in vivo. To study DDR2 contribution to tumor cell colonization and growth we utilized our experimental model of intrasplenically inoculated cancer cells $(18,19)$. We also used a melanoma cell line to test DDR2 implication in metastatic development because melanoma is one of the most frequent primary tumors that metastasize to the liver. We use the siRNA technology to partially silence the DDR2 protein expression in the tumor cells and then injected them intrasplenically to compare their ability to metastasize to the liver with that of non-transfected and mock transfected tumor cells. The silencing of DDR2 protein in the tumor cells produced a

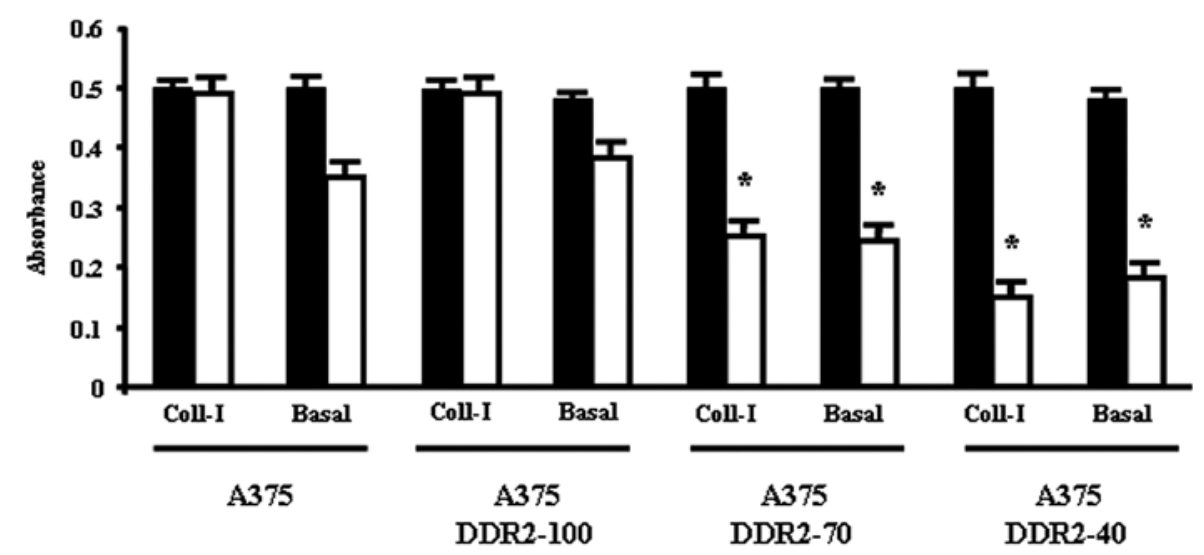

Figure 8. JNK pathway is involved in DDR2 signalling in A375 human melanoma cell lines. We performed an ELISA to measure total JNK (black bars) and phosphorilated JNK (white bars) proteins in A375 clones maintained under basal conditions or in the presence of collagen type I. Statistically significant results ${ }^{*} \mathrm{p}<0.05$ 
statistically significant tumor progression delay that correlated with DDR2 protein silencing levels. Higher DDR2 silencing correlated with less liver area occupied by the tumor, which shows a direct relationship between the presence of DDR2 and liver metastasis progression.

Our previous studies on hepatic stellate cells and skin fibroblasts during heptic and skin injury, respectively, revealed that DDR2 signaling regulates key aspects of wound-repair such as MMP2 synthesis, cell proliferation and chemotactic invasion $(11,12,17)$. MMPs are the major protein source implicated in extracellular matrix degradation in healthy and diseased tissues $(17,11,24)$. We used siDDR2 against DDR2 expression with in vitro MMP2/9 secretion, cell proliferation and cell migration $(20,21)$ because they are also key prometastatic characteristics of tumor cells (22). In vitro activation of DDR2 induces the expression of MMP2 (11). MMP2 together with MMP9 and MMP1 $(24,25)$, are the main enzymes for extracellular matrix remodelling during tissue repair (23). MMPs have also been described in metastatic progression because their activity is required for cancer cell motility $(24,25)$. MMPs are also involved in other cell-mediated events during metastatic progression. For example, the mitogenic response of some growth factors is regulated by MMPs, which control the availability of the receptor (24). In addition, MMP degradation of the extracellular matrix allows some signalling cascades to interconnect (22). In this study, we showed that downregulation of DDR2 expression correlates with reduction of MMP9 and MMP2 activity, and this reduction was more pronounced when the cells had been previously activated with collagen. This may indicate that DDR2 signalling in response to collagen is a key event in tumor production of MMP9 and MMP2.

MMP activity has been related with tumor cell proliferation and migration (26). Proliferation is an important factor in metastatic progression. Faster in vitro tumor growth directly correlates with its experimental metastatic process. Thus, we measured proliferation and migration in three human tumor cell lines from three different tissue origin: skin, liver and colon and submitted them to partial transient DDR2 silencing. Proliferation was diminished in cells with DDR2 expression partially silenced, and this reduction was more pronounced when cells were cultured in the presence of DDR2 ligand, collagen type I. Therefore, DDR2 expression levels may be a marker of tumor aggressiveness.

Tumor migration ability is directly involved in the first critical steps of metastasis, such as proteolysis of the extracellular matrix together with invasion through the endothelial barrier (27), and further implantation and growth in the metastized organ. Silencing of DDR2 protein expression reduced tumor migration. We demonstrated that DDR2 downregulation reduced drastically tumor cell migration rate in response to DDR2 ligand, fibrillar collagen type I.

We also demonstrated that DDR2 signals via JNK in A375 melanoma cells. PKC, FAK, MEK, MAPK and JNK pathways downstream of DDR2 have been described (28-32). We focused on the study of JNK because JNK phosphorylation has been implicated in the expression of several types of MMP, such as MMP1, MMP2 and MMP9 (33-35), and in cell proliferation (34). Silencing of DDR2 receptor expression correlated with decreased phosphorylated JNK beyond the control levels, suggesting a DDR2 autophosphorylation event independent of the ligand interaction.

In summary, this study provides evidence for DDR2 expression and functionality in several human cancer cell lines, such as SK-HEP hepatocarcinoma, HT-29 coloncarcinoma and A375 melanoma. Furthermore, in vivo experiment showed a direct correlation between DDR2 in human A375 cells and their experimental liver metastasis ability. The underlying mechanism involves regulation of MMP9 and MMP2 activity, cell proliferation and migration via JNK signaling. Further studies are required to confirm DDR2 as a malignancy marker or therapeutic target.

\section{References}

1. Augsburger JJ, Correa ZM and Shaih AH: Effectiveness of treatments for metastatic uveal melanoma. Am J Ophthalmol 148: 119-127, 2009.

2. Nemoto T, Ohashi K, Akashi T, Johnson JD and Hirokawa K: Overexpression of protein tyrosine kinases in human esophageal cancer. Pathobiology 65: 195-203, 1997.

3. Blume-Jensen P and Hunter T: Oncogenic kinase signalling. Nature 411: 355-365, 2001.

4. Schlessinger J and Lemmon MA: SH2 and PTB domains in tyrosine kinase signaling. Sci STKE re12, 2003.

5. Posner BI and Laporte SA: Cellular signaling: Peptide hormones and growth factors. Prog Brain Res 181: 1-16, 2010.

6. Shrivastava A, Radziejewski C, Campbell E, et al: An orphan receptor tyrosine kinase family whose members serve as nonintegrin collagen receptors. Mol Cell 1: 25-34, 1998.

7. Yan SH, Baek HA, Lee HJ, et al: Discoidin domain receptor 1 is associated with poor prognosis of non-small cell lung carcinomas. Oncol Rep 24: 311-319, 2010.

8. Day E, Water B, Spiegel K, et al: Inhibition of collagen-induced discoidin domain receptor 1 and 2 activation by imatinib, nilotinib and dasatinib. Eur J Pharmacol 599: 44-53, 2008.

9. Park HS, Kim KR and Lee HJ: Overexpression of discoidin domain receptor 1 increases the migration and invasion of hepatocellular carcinoma cells in association with matrix metalloproteinase. Oncol Rep 18: 1435-1441, 2007.

10. Lai $\mathrm{C}$ and Lemke G: Structure and expression of the Tyro 10 receptor tyrosine kinase. Oncogene 9: 877-883, 1994.

11. Olaso E, Ikeda K, Eng FJ, Xu L, Wang L, Lin H and Friedman SL: DDR2 receptor promotes MMP2-mediated proliferation and invasion by hepatic stellate cells. J Clin Invest 108: 1369-1378, 2001.

12. Olaso E, Labrador JP, Wang LH, Ikeda K, Eng FJ, Klein R, Lovett DH, Lin H and Friedman SL: Discoidin domain receptor 2 regulates fibroblast proliferation and migration through the extracellular matrix in association with transcriptional activation of matrix metalloproteinase-2. J Biol Chem 277: 3606-3613, 2002.

13. Vogel W, Gish GD, Alves F and Pawson T: The discoidin domain receptor tyrosine kinases are activated by collagen. Mol Cell 1: 13-23, 1997.

14. Olaso E, Santisteban A, Bidaurrazaga J, et al: Tumor dependent activation of rodent hepatic stellate cells during experimental melanoma metastasis. Hepatology 26: 634-642, 1997.

15. Mossman T: Rapid colorimetric assay for cellular growth and survival: application to proliferation and cytotoxicity assays. J Immunol 65: 55-63, 1983.

16. Cao Q, Mak KM and Lieber CS: Leptin represses matrix metallloproteinase-1 gene expression in LX2 human hepatic stellate cells. J Hepatol 46: 124-133, 2007.

17. Labrador JP, Azcoitia V, Tuckerman J, Lin C, Olaso E, Mañes S, Bruckner K, Goergen JL, Lemke G, Yancopoulus G, Angel P and Klein R: The collagen receptor DDR2 regulates proliferation and its elimination leads to dwarfism. EMBO Rep 2: 446-452, 2010.

18. Olaso E, Salado C, Egilegor E, et al: Proangiogenic role of tumor-activated hepatic stellate cells in experimental melanoma metastasis. Hepatology 37: 674-685, 2003.

19. Solaun MS, Mendoza L, De Luca M, et al: Endostatin inhibits murine colon carcinoma sinusoidal-type metastases by preferential targeting of hepatic sinusoidal endothelium. Hepatology 35: 1104-1116, 2002. 
20. Suojanen J, Salo T, Koivunen E, Sorsa T and Pirilä E: A novel and selective membrane type-1 matrix metalloproteinase (MT1-MMP) inhibitor reduces cancer cell motility and tumor growth. Cancer Biol Ther 19: 8-24, 2009.

21. Werb Z: ECM and cell surface proteolysis: regulating cellular ecology. Cell 14;91: 439-442, 1997.

22. Diamantopoulos N, Boutis AL, Koratzis I, Andreadis C, Galaktidou G, Mouratidou D and Kortsaris A: Matrix metalloproteinases and proangiogenic factors in testicular germ cell tumors. J BUON 15: 116-121, 2010.

23. Vogel WF, Abdulhussein R and Ford CE: Sensing extracellular matrix: an update on discoidin domain receptor function. Cell Signal 18: 1108-1116, 2006

24. Zhao XL, Sun T, Che N, Sun D, Zhao N, Dong XY, Gua Q, Yao Z and Sun BC: Promotion of hepatocellular carcinoma metastasis through matrix metalloproteinase activation by epithelial-mesenchymal transition regulator twist1. J Cell Mol Med 15: 691-700, 2011

25. Dong Z, Ghabrial M, Katar M, Fridman R and Berks RS: Membrane-type matrix metalloproteinases in mice intracorneally infected with Pseudomonas aeruginosa. Invest Ophthalmol Vis Sci 41: 4189-4194, 2000.

26. Yoon JH, Kim SA, Kim JI, Park JH, Ahn SG and Yoon JH: Inhibition of invasion and migration of salivary gland adenocarcinoma cells by 5 '-nitro-indirubinoxime (5'-NIO). Head Neck 32 619-625, 2010.

27. Fidler IJ: The pathogenesis of cancer metastasis: The 'seed and soil' hypothesis revised. Nat Rev Cancer 3: 453-458, 2003.

28. Vonk LA, Donkbi BZ, Huang C, et al: Collagen induced expression of collagenase 3 by primary chondrocytes is mediated by integrin alpha 1 and discoidin domain receptor 2: a protein kinase C-dependent pathway. Rheumatology: Nov. 12, 2010 (Epub ahead of print)
29. Lin KL, Chan CH, Hsie SC, et al: Transcriptional upregulation of DDR 2 by ATF4 facilitates osteoblastic differentiation through p38 MAPK-mediated Runx-2 activation. J Bone Miner Res 25: 2489-2503, 2010

30. Fuchs SY, Adler V, Pincus MR and Ronai Z: MEKK1/JNK signaling stabilizes and activates p53. Proc Natl Acad Sci USA 95: 10541-10546, 1998.

31. Lim CP and Cao X: Regulation of Stat 3 Activation by MEK Kinase. J Biol Chem 276: 21004-21011, 2001.

32. Auer KL, Contessa JB, Pirolla LR, et al: The Ras/Rac1/Cdc42/ SEK/JNK/c-Jun cascade is a key pathway by which agonists stimulate DNA synthesis in primary cultures of rat hepatocytes. Mol Biol Cell 9: 561-573, 1998.

33. Endo H, Watanabe T, Sugioka Y, Niioka M, Inagaki Y and Okazaki I: Activation of two distinct MAPK pathways governs constitutive expression of matrix metalloproteinase-1 in human pancreatic cancer cell lines. Int J Oncol 35: 1237-1245, 2009.

34. Hsien HL, Wang HH, Wu WB, Chu PJ and Yang CM: Transforming growth factor- $\beta 1$ induces matrix metalloproteinase and cell migration in astrocytes: roles of ROS-dependent ERK- and JNK-NF-k $\beta$ pathways. J Neuroinflammation 6: 7-18, 2010.

35. Ye Y and Dan Z: All-trans retinoic acid diminishes collagen production in a hepatic stellate cell line via suppression of actine protein-1 and c-Jun $\mathrm{N}$ terminal kinase signal. J Huazhong Univ Sci Technolog Med Sci 30: 726-733, 2010.

36. Hui L, Zatloukal K, Scheuch H, Stepniak E and Erwin FW: Proliferation of human HCC cells and chemically induced mouse liver cancers requires JNK1-dependent p21 downregulation. J Clin Invest 118: 3943-3953, 2008. 